\title{
Identifying the host galaxy of the short GRB 100628A ${ }^{\star}$
}

\author{
A. Nicuesa Guelbenzu ${ }^{1}$, S. Klose ${ }^{1}$, E. Palazzi ${ }^{2}$, J. Greiner ${ }^{3}$, M. J. Michałowski ${ }^{4}$, D. A. Kann ${ }^{1}$, L. K. Hunt ${ }^{5}$,

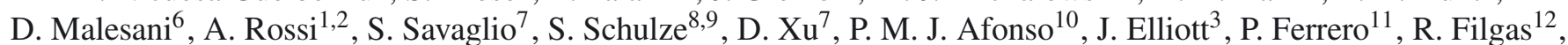 \\ D. H. Hartmann ${ }^{13}$, T. Krühler ${ }^{14}$, F. Knust ${ }^{3}$, N. Masetti ${ }^{2,15}$, F. Olivares E. ${ }^{15}$, A. Rau ${ }^{3}$, P. Schady ${ }^{3}$, S. Schmidl ${ }^{1}$, \\ M. Tanga ${ }^{3}$, A. C. Updike ${ }^{16}$, and K. Varela ${ }^{3}$
}

1 Thüringer Landessternwarte Tautenburg, Sternwarte 5, 07778 Tautenburg, Germany e-mail: ana@tls-tautenburg.de

2 INAF-IASF Bologna, via Gobetti 101, 40129 Bologna, Italy

3 Max-Planck-Institut für Extraterrestrische Physik, Giessenbachstraße 1, 85748 Garching, Germany

${ }^{4}$ Scottish Universities Physics Alliance for Astronomy, University of Edinburgh, Royal Observatory, Edinburgh, EH9 3HJ, UK

5 INAF-Osservatorio Astrofisico di Arcetri, Largo E. Fermi 5, 50125 Firenze, Italy

${ }^{6}$ Dark Cosmology Centre, Niels Bohr Institute, University of Copenhagen, Juliane Maries Vej 30, 2100 Copenhagen, Denmark

7 Physics Department, University of Calabria, via P. Bucci, 87036 Arcavacata di Rende, Italy

8 Instituto de Astrofísica, Facultad de Física, Pontificia Universidad Católica de Chile, 306, Santiago 22, Chile

9 Millennium Institute of Astrophysics, Vicuña Mackenna 4860, 7820436 Macul, Santiago, Chile

10 American River College, Physics and Astronomy Dpt., 4700 College Oak Drive, Sacramento, CA 95841, USA

11 Instituto de Astrofísica de Andalucía, Consejo Superior de Investigaciones Científicas (IAA-CSIC), Glorieta de la Astronomía s/n, 18008 Granada, Spain

12 Institute of Experimental and Applied Physics, Czech Technical University in Prague, Horská 3a/22, 12800 Prague, Czech Republic

13 Department of Physics and Astronomy, Clemson University, Clemson, SC 29634, USA

14 European Southern Observatory, Alonso de Córdova 3107, 19001 Vitacura Casilla, Santiago 19, Chile

15 Departamento de Ciencias Fisicas, Universidad Andres Bello, Avda. Republica 252, Santiago, Chile

16 Department of Chemistry and Physics, Roger Williams University, One Old Ferry Rd, Bristol, RI, 0209, USA

Received 15 October 2014 / Accepted 15 August 2015

\section{ABSTRACT}

\begin{abstract}
We report on the results of a comprehensive observing campaign to reveal the host galaxy of the short GRB 100628A. This burst was followed by a faint X-ray afterglow but no optical counterpart was discovered. However, inside the X-ray error circle a potential host galaxy at a redshift of $z=0.102$ was soon reported in the literature. If this system is the host, then GRB 100628A was the cosmologically most nearby unambiguous short burst with a measured redshift so far. We used the multi-colour imager GROND at the ESO/La Silla MPG 2.2 m telescope, ESO/VLT spectroscopy, and deep Australia Telescope Compact Array (ATCA) radiocontinuum observations together with publicly available Gemini imaging data to study the putative host and the galaxies in the field of GRB 100628A. We confirm that inside the X-ray error circle the most probable host-galaxy candidate is the morphologically disturbed, interacting galaxy system at $z=0.102$. The interacting galaxies are connected by a several kpc long tidal stream, which our VLT/FORS2 spectroscopy reveals strong emission lines of [O II], [O III], $\mathrm{H} \alpha$ and $\mathrm{H} \beta$, characteristic for the class of extreme emissionline galaxies and indicative of ongoing star formation. The latter leaves open the possibility that the GRB progenitor was a member of a young stellar population. However, we indentify a second host-galaxy candidate slightly outside the X-ray error circle. It is a radiobright, luminous elliptical galaxy at a redshift $z=0.311$. With a $K$-band luminosity of $2 \times 10^{11} L_{\odot}$ this galaxy resembles the probable giant elliptical host of the first well-localized short burst, GRB 050509B. If this is the host, then the progenitor of GRB 100628A was a member of an old stellar population.
\end{abstract}

Key words. gamma-ray burst: individual: 100628A

\section{Introduction}

Gamma-ray bursts (GRBs) are divided into long-soft and shorthard categories (Mazets \& Golenetskii 1981; Norris et al. 1984), with the borderline at two seconds duration (Kouveliotou et al. 1993). It is commonly accepted that long GRBs are linked to the collapse of very massives stars (see Woosley \& Bloom 2006; Hjorth \& Bloom 2012, for reviews), while short GRBs are

* Based on observations collected at the Very Large Telescope of the European Southern Observatory, Paranal, Chile (ESO programme 087.D-0503 and 290.D-5194; PI: A. Nicuesa Guelbenzu; 090.A-0825; PI: D. Malesani), GROND (PI: J. Greiner), and ATCA (Program C2840; PI: A. Nicuesa Guelbenzu). Other observations are obtained from the Gemini and WISE Archive Facilities. thought to originate from stellar mergers of two neutron stars (NSs) or a NS and a black hole (see, e.g., Nakar 2007; Lee \& Ramirez-Ruiz 2007, for reviews). Short GRBs occur in all morphological types of galaxies, from elliptical to irregular, in clusters and in the field, with observed redshifts ranging from $z \sim 0.1$ to about 1. Among the ensemble of about 30 identified and wellstudied hosts of short bursts (for a review, see Berger 2014), less than $1 / 3$ are elliptical galaxies. All others show signs of starforming activity, and at least three of these are even starburst galaxies with star formation rates of the order of $100 M_{\odot} \mathrm{yr}^{-1}$ (Perley et al. 2012; Berger et al. 2013; Nicuesa Guelbenzu et al. 2014).

While in elliptical galaxies these merging binary stars might be members of an old stellar population (at least for low 
redshifts), in star-forming galaxies such stars could also be young. In fact, stellar evolution models suggest a broad range in the ages of merging binaries, reaching from billions down to millions of years (Belczynski et al. 2006). Unfortunately, in most cases the explosion sites of short GRBs in their cosmologically remote hosts remain unidentified or cannot be spatially resolved. Nevertheless, estimates of the characteristic ages of short-GRB progenitors can be obtained by analysing the stellar ages and masses of their host galaxies (Leibler \& Berger 2010). More data are needed here to improve the statistics and, consequently, every identified host galaxy counts. The observational progress is slow, since on average no more than three to five short-GRB host galaxies are identified per year (e.g., Kann et al. 2011; Nicuesa Guelbenzu et al. 2012; D’Avanzo et al. 2014).

GRB $100628 \mathrm{~A}$ is a member of the group of short bursts currently without a well-studied host galaxy. The burst triggered the Swift/Burst Alert Telescope (BAT; Barthelmy et al. 2005) as well as the INTEGRAL satellite (Beckmann et al. 2010). In the BAT energy window $(15-350 \mathrm{keV})$ the burst had a duration of $T_{90}=0.036 \pm 0.009 \mathrm{~s}$ (Immler et al. 2010), and its X-ray afterglow was very faint. This led to two revisions of the initially reported (Starling \& Immler 2010) Swift/X-ray telescope (XRT; Burrows et al. 2005) position. The first revised error circle (Starling et al. 2010a) encompasses a source that, in fact, was later found to be an active galactic nucleus (AGN; Berger 2010a), most likely unrelated to the GRB. The final revised Xray error circle is located at coordinates RA, Dec $(\mathrm{J} 2000)=$ 15:03:52.41, -31:39:30.2 and has an error radius of $7^{\prime \prime}$ (90\% c.1., Starling et al. 2010b). No UV/optical/near-infrared afterglow was detected, neither by Swift/Ultra-Violet/Optical Telescope (UVOT; Roming et al. 2005) nor by ground-based observatories (Immler 2010; Burenin et al. 2010; Berger et al. 2010b,a; Berger 2010b; Suzuki et al. 2010; Levan et al. 2010; Nicuesa Guelbenzu et al. 2012). No radio observations of the afterglow were reported. However, it was soon found that an interacting galaxy system is present inside the final XRT error circle at a redshift of $z=0.102$ (Berger 2010b; Cenko et al. 2010). If this interacting galaxy system were the host of GRB 100628A this would be the nearest short burst with a measured distance thus far. Motivated by this fact, we report on the results of a comprehensive multi-wavelength observing campaign to study this galaxy, including imaging with GROND on La Silla, spectroscopy with the ESO/VLT, and deep ATCA radio-continuum observations.

Throughout the paper, we adopt a $\Lambda$ CDM cosmology $\left(\Omega_{\mathrm{M}}=\right.$ 0.27, $\Omega_{\Lambda}=0.73, H_{0}=71 \mathrm{~km} \mathrm{~s}^{-1} \mathrm{Mpc}^{-1}$; Spergel et al. 2003).

\section{Observations and data reduction}

\subsection{Imaging}

Imaging data of the field were obtained at the time of the burst (Updike et al. 2010) using the optical/near-infrared sevenchannel imager GROND (Greiner et al. 2008) mounted at the MPG 2.2 m telescope at ESO La Silla Observatory (Chile). In addition, we made use of publicly available Gemini archival $i$-band data, which are deeper than the GROND images (Levan et al. 2010; Program IDs GN-2010A-Q-7, PI: N. Tanvir and GS2010A-Q-5, PI: D. Fox). The GROND optical data were calibrated against the standard star field SDSS J141826.3-02333. The Gemini $i$-band data were calibrated based on the GROND $i^{\prime}$-band data. For the near-infrared (NIR) bands, photometric calibration was performed against the 2MASS catalogue (Skrutskie et al. 2006). Data were reduced in a standard fashion using IRAF tasks ${ }^{1}$ implemented in the pipeline written to reduce GROND data (Krühler et al. 2008; Küpcü Yoldaş et al. 2008).

\subsection{VLT spectroscopy}

We performed VLT/FORS2 long-slit spectroscopy on the field of GRB 100628A using two different slit positions to cover the maximum number of objects inside and close to the XRT error circle (altogether three visits; Program IDs 087.D-0503 and 290.D-5194, PI: A. Nicuesa Guelbenzu; Table 2; Fig. 2). All observations were executed at an airmass $<1.1$. In the first two runs we used a slit width of $1{ }^{\prime \prime} 0$, while in the third run the slit width was 1 ". 3 . In all runs we used the $300 \mathrm{~V}+10$ grism (dispersion $112 \AA \mathrm{mm}^{-1}, 1.68 \AA \mathrm{pixel}^{-1}$, resolution $R=440$ at $590 \mathrm{~nm}^{2}$ ); in the third run we used it in combination with the GG 435 order separation filter. We applied cosmic ray, flatfield, and bias correction during the data reduction in the usual way with IRAF (Tody 1993). Wavelength calibration was performed relative to $\mathrm{HgCdHe}+\mathrm{Ar}$ calibration lamps. The spectrophotometric standard stars LTT 7379 (April 2011; Table 2), LTT 7987 (May 2011), and LTT 6248 (April 2013) were observed to fluxcalibrate the different spectra.

The VLT/X-Shooter observed the field in 2013 (Program ID 090.A-0825, PI D. Malesani; Table 2). The data were reduced in a standard fashion using the X-Shooter pipeline v2.5 (Goldoni et al. 2006). To extract the one-dimensional spectra, we used a customized tool that adopts the optimal extraction algorithm (Horne 1986). The spectrum was flux-calibrated with the spectrophotometric standard star LTT 3218 and all wavelengths were transformed to vacuum wavelengths. No telluric correction was applied, as it had no implications for our analysis.

\subsection{ATCA radio observations}

Radio-continuum observations of the field of GRB 100628A were performed in July 2013 in the 5.5 and $9.0 \mathrm{GHz}$ bands (corresponding to 6 and $3 \mathrm{~cm}$ ) with the Australia Telescope Compact Array (ATCA) using the upgraded Compact Array Broadband Backend (CABB) detector (Wilson et al. 2011) and all six 22-m antennae with the $6 \mathrm{~km}$ baseline (program ID: C2840, PI: A. Nicuesa Guelbenzu; Table 2). The width of the primary beam is $9 \operatorname{arcmin}$ (at $5.5 \mathrm{GHz}$ ) and $5 \operatorname{arcmin}(9.0 \mathrm{GHz}$ ), respectively (ATCA users guide ${ }^{3}$ ), and the synthesized beam sizes were $\sim 2$ '. $0 \times 44^{\prime \prime} .2$ and $\sim 1^{\prime \prime} .2 \times 2$ '. 6 , at 6 and $3 \mathrm{~cm}$, respectively. The CABB integrates in both bands simultaneously with 2048 channels, beginning at 4.476 and $7.976 \mathrm{GHz}$, respectively, with an increment of $1 \mathrm{MHz}$. Bandpass and flux calibration was done by observing PKS B1934-638 at the end of the observing run. Phase calibration was performed by observing the source 1451375 for $7 \mathrm{~min}$ before and after each integration on target for $53 \mathrm{~min}$. Altogether ten such 1-h cycles were executed in this way, covering for our target nearly a complete $(u, v)$ plane.

We reduced the data with the software package MIRIAD for ATCA radio interferometry ${ }^{4}$, following the procedure outlined in Nicuesa Guelbenzu et al. (2014). We cleaned all of the data from radio frequency interference (RFI) using the pgflag routine. The effective bandwidth was thus reduced by $14.4 \%$ to $1753 \mathrm{MHz}$. Deconvolution was performed using the mfclean

\footnotetext{
http://iraf.noao.edu

2 See the FORS2 User Manual dated 26/02/2013, page 12.

3 http://www.narrabri.atnf.csiro.au/observing/users_ guide/ html/new_atug.pdf

4 http://www.atnf.csiro.au/computing/software/miriad
} 


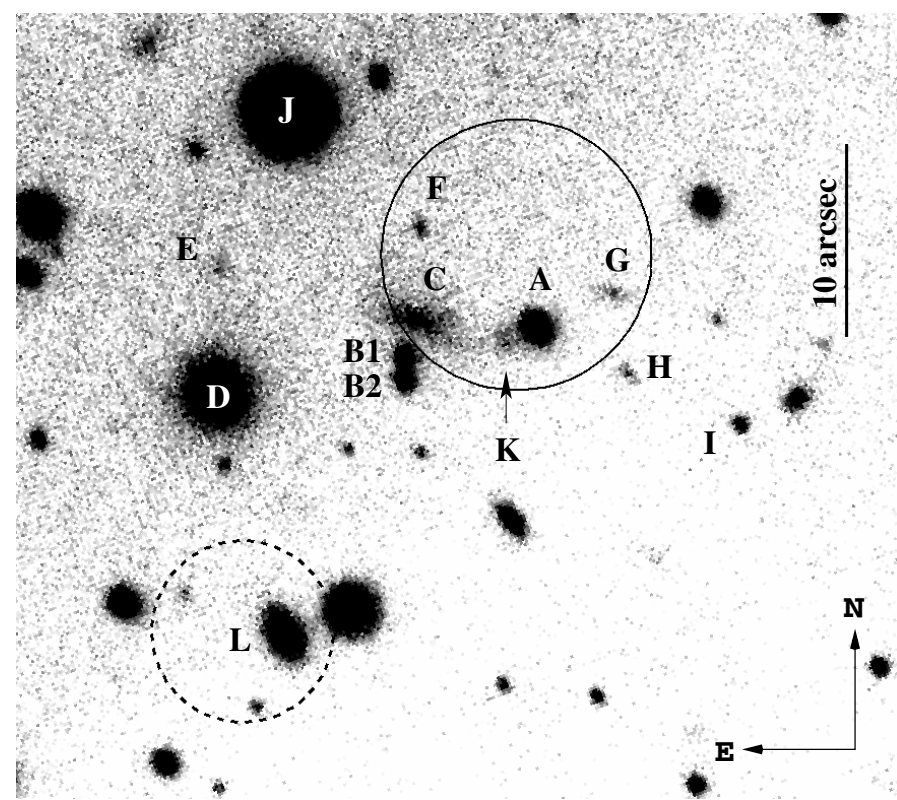

Fig. 1. Gemini-South/GMOS-S $i$-band image of the field of the short GRB 100628A. Also shown is the first revised (radius 4".7; bottom left, broken line; Starling et al. 2010a) and the final Swift/XRT error circle (radius 7"; solid line; Starling et al. 2010b). Object L is the galaxy detected by Chandra X-ray observations (Berger 2010a). Because of a slight tracking error of most of the individual images, stars appear slightly elongated.

procedure. Finally, calibrated and RFI-cleaned visibilities were Fourier transformed using the "robust" weighting option, varying this parameter between "natural" and "uniform" weighting and selecting the weighting that gave the best result, reaching an rms value of $6 \mu \mathrm{Jy}$ at $5.5 \mathrm{GHz}$ and $7 \mu \mathrm{Jy}$ at $9.0 \mathrm{GHz}$. Flux measurements were done using the imfit task by fitting a twodimensional Gaussian profile.

\section{Results and discussion}

\subsection{Inside the XRT error circle: Galaxy $C$}

In the Gemini $i$-band image, inside the final 7 arcsec (radius) XRT error circle, we identify five objects, labelled A, C, F, G, and K (Fig. 1). The VLT/FORS2 spectra revealed that object A is a foreground star, while the VLT/X-Shooter spectrum showed that object $\mathrm{F}$ is a galaxy at a redshift of $z=0.406$. Galaxy $\mathrm{G}$ is a faint, but clearly extended object, which was not covered completely by the FORS 2 slit, and no trace of this object could be identified in the $2 \mathrm{D}$ spectrum. Thus, its redshift remains unknown.

The most prominent galaxy inside the error circle is object $\mathrm{C}$. This object distinguished by its relatively large angular size, its relatively bright apparent magnitude (observed $\mathrm{AB}$ magnitude $i^{\prime} \sim 21.5$ ), and its disturbed morphology.

We adopt the methodology of Bloom et al. (2002) and the $i$-band galaxy number counts of Henriques et al. (2012) to quantify the probability that this galaxy is a chance superposition. We find a probability $p$ of 0.07 that a galaxy with $i(\mathrm{AB}) \leq 21.5$ mag lies within the aforementioned XRT error circle. The corresponding probabilities for the fainter galaxies $\mathrm{G}$ and $\mathrm{F}$ (Table 1) are notably larger ( 0.64 and 0.77 , respectively). In conclusion, among all galaxies visible inside the XRT error circle, the small $p$-value makes object $\mathrm{C}$ the most probable host-galaxy candidate.
Table 1. Objects inside and close to the XRT error circle of GRB 100628A.

\begin{tabular}{lllrl}
\hline \hline Object & RA (J2000) & Dec & $i_{\mathrm{AB}}$ & Redshift $z$ \\
\hline A & $15: 03: 52.34$ & $-31: 39: 33.9$ & 20.8 & K star \\
B1 & $15: 03: 52.86$ & $-31: 39: 35.4$ & 22.1 & M star \\
B2 & $15: 03: 52.86$ & $-31: 39: 36.7$ & 22.3 & star \\
C & $15: 03: 52.81$ & $-31: 39: 33.6$ & $\sim 21.5$ & 0.102 \\
D & $15: 03: 53.598$ & $-31: 39: 37.89$ & 20.2 & 0.311 \\
E & $15: 03: 53.61$ & $-31: 39: 30.9$ & 24.5 & 0.099 \\
F & $15: 03: 52.80$ & $-31: 39: 28.8$ & 24.1 & 0.406 \\
G & $15: 03: 52.02$ & $-31: 39: 32.3$ & 23.8 & galaxy \\
H & $15: 03: 51.96$ & $-31: 39: 36.3$ & 23.7 & galaxy \\
I & $15: 03: 51.50$ & $-31: 39: 38.9$ & 23.1 & 0.179 \\
J & $15: 03: 53.36$ & $-31: 39: 22.6$ & 17.6 & star \\
K & $15: 03: 52.45$ & $-31: 39: 34.7$ & 23.8 & 0.102 \\
L & $15: 03: 53.35$ & $-31: 39: 49.9$ & 20.5 & galaxy \\
\hline
\end{tabular}

Notes. For object $\mathrm{D}$, the coordinates are based on the radio data at 9.0 GHz. Redshifts, when measured, are given with an accuracy of \pm 0.001 . $i$-band $\mathrm{AB}$ magnitudes are observed magnitudes and were measured on the combined Gemini-S/GMOS frames.

Table 2. Log of the observations.

\begin{tabular}{lccc}
\hline \hline Date & Telescope & Instrument & Exposure \\
\hline 2010 Jun. 29 & $2.2 \mathrm{~m}$ & GROND $/ g^{\prime} r^{\prime} i^{\prime} z^{\prime} J H K_{\mathrm{s}}$ & $1.5 \mathrm{~h}$ \\
2010 Jun. 29 & Gemini-S & GMOS-S $/$ band & $12 \times 120 \mathrm{~s}$ \\
2011 Apr. 11 & VLT/UT1 & FORS2/GRIS 300V+10 & $2 \times 1240 \mathrm{~s}$ \\
2011 May 26 & VLT/UT1 & FORS2/GRIS 300V+10 & $2 \times 1240 \mathrm{~s}$ \\
2013 Mar. 20 & VLT/UT2 & X-Shooter & $4 \times 940 \mathrm{~s}$ \\
2013 Apr. 03 & VLT/UT1 & FORS2/GRIS 300V+10 & $2 \times 1240 \mathrm{~s}$ \\
2013 Jul. 23 & ATCA & CABB & $11 \mathrm{~h}$ \\
\hline
\end{tabular}

On the Gemini $i$-band image, galaxy $\mathrm{C}$ has a diameter (major axis) of about $5^{\prime \prime}$, at $z=0.102$ equivalent to $\sim 10 \mathrm{kpc}^{5}$. The light bridge that originates in $\mathrm{C}$ and extends in the western direction towards the foreground star A (including object K; Fig. 1) is most likely a tidal tail. Its length, as estimated from the Gemini $i$-band image, is about 3 .' 6 , corresponding to $6.5 \mathrm{kpc}$. Our spectra show that object $\mathrm{K}$ is also at $z=0.102$ (Table 3). In order to reveal its detailed morphology, we subtracted the point-spread function (PSF) of star A, but could not gain additional information about the nature of object K. Probably, this is either the interacting partner of galaxy $\mathrm{C}$ or simply the brightest knot in the tidal tail originating from $\mathrm{C}$. We also performed PSF subtraction of the stars B1 and B2, but again did not reveal new details about the galaxy-C complex. There is also a hint of a fainter tidal tail of similar length as the east-west tail, extending from $\mathrm{C}$ towards galaxy $\mathrm{F}$, though physically unrelated to $\mathrm{F}$ since the galaxies lie at very different redshifts. Finally, also galaxy $\mathrm{E}$ is excluded as the interacting partner of C. Even though this redshift is close to that of galaxy $\mathrm{C}$, the difference in luminosity distance is $\sim 15 \mathrm{Mpc}$.

Following Nicuesa Guelbenzu et al. (2012), using the GROND data, we produced a white-band image in the optical $\left(g^{\prime} r^{\prime} i^{\prime} z^{\prime}\right)$ and in the NIR $\left(J H K_{\mathrm{s}}\right)$ bands to study the complex morphology of galaxy $\mathrm{C}$. These images revealed two additional faint objects in the western outskirts of galaxy C (labelled \#x and \#y) and an NIR-bright object close to A (Fig. 3). Object \#y

\footnotetext{
5 At this redshift the luminosity distance is $1.43 \times 10^{27} \mathrm{~cm}(465 \mathrm{Mpc})$, $m-M=38.34 \mathrm{mag}$, the look-back time is $1.31 \mathrm{Gyr}$, and $1^{\prime \prime}$ corresponds to $1.86 \mathrm{kpc}$.
} 


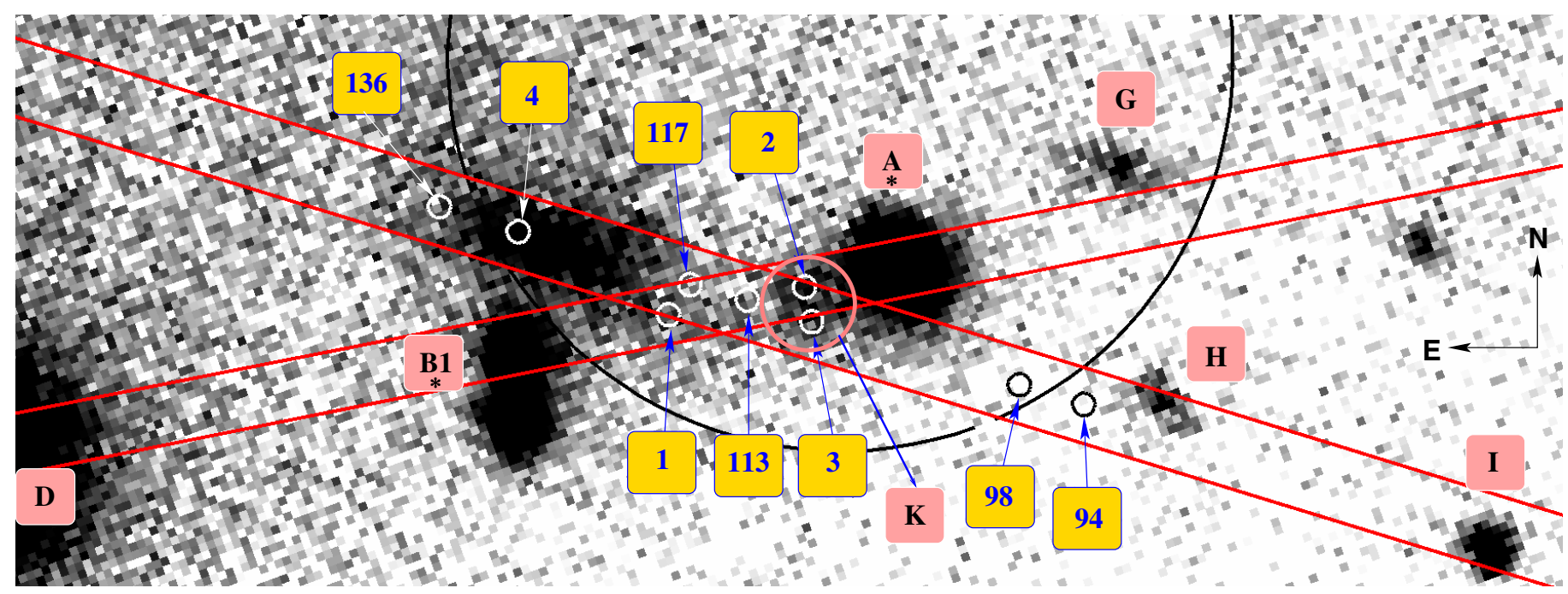

Fig. 2. Sketch of the two different slit positions and slit widths of our three VLT/FORS2 visits of the field of GRB 100628A, overlaid onto the Gemini $i$-band image. Positions with strong emission lines in the 2D spectra (in the following called blobs) are labelled with small circles and numbered (numbers larger than 4 refer to the pixel coordinate along the corresponding slit). Blobs 1, 113, and 2 are seen in the 2D spectrum with the slit oriented from south-east to north-west, i.e., when the slit covered objects B1 and A. All other blobs show up in the 2D spectrum with the slit oriented in the other direction. Blobs 1 and 117 might belong to the same emission-line region in galaxy $\mathrm{C}$ (see Fig. 1). The same holds for blobs 2 and 3, which might belong to object K. Regions shown in yellow are at a common redshift $z=0.102 \pm 0.001$, the redshift of galaxy C. Except for object K, all objects shown in pink are either foreground stars (A, B1) or galaxies (D, G, H, I). Object K could be the interacting partner of C.

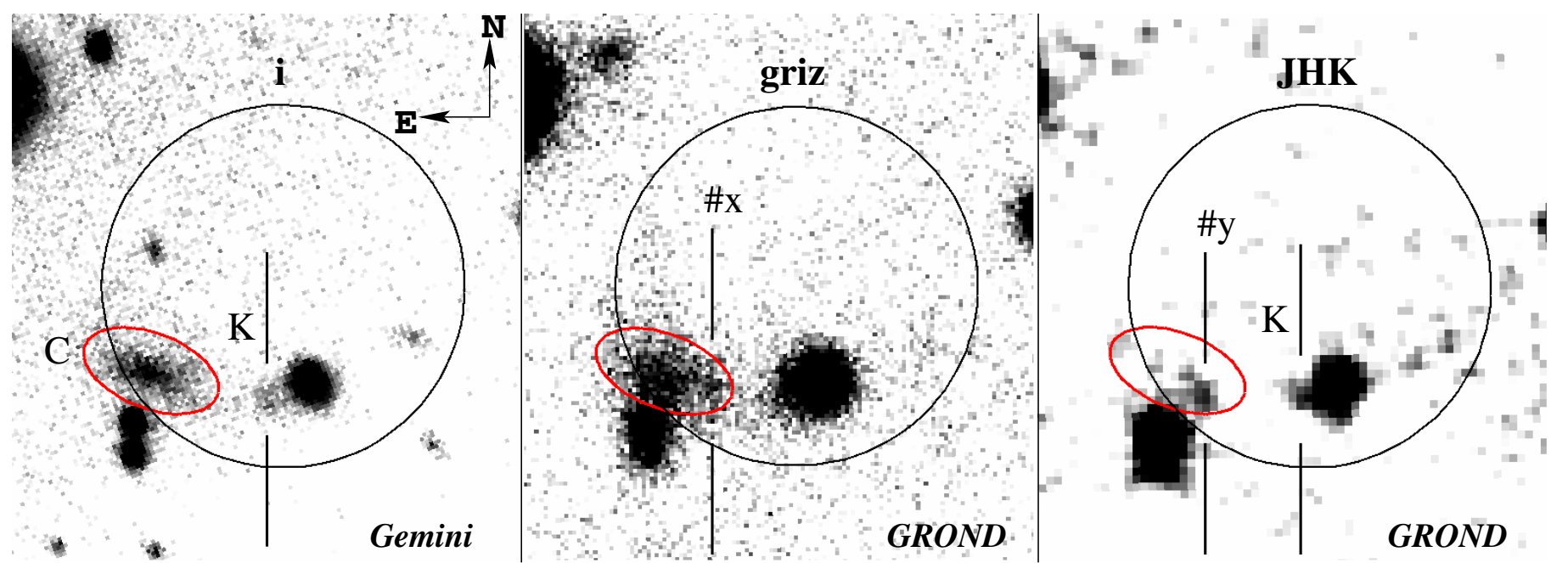

Fig. 3. Gemini $i$-band as well as GROND combined $g^{\prime} r^{\prime} i^{\prime} z^{\prime}$ and $J H K_{\mathrm{s}}$ white-band images. Object K is clearly detected in the NIR. In addition, the GROND images reveal two more blobs designated \#x and \#y. The former is only detected in the optical, the latter only in the NIR. Within the astrometric $3 \sigma$ error, \#y could be the NIR counterpart of \#x. Also shown is the XRT error circle (radius 7") as well as an ellipse circumscribing galaxy $\mathrm{C}$ just to guide the eye $\left(2{ }^{\prime \prime} 5 \times 1\right.$ 1." $\left.^{\prime}\right)$. North is up and east is to the left.

Table 3. Observed line strengths of nine blobs detected with emission lines in the VLT/FORS2 2D spectra.

\begin{tabular}{rccccccccc}
\hline \hline$\#$ & $\begin{array}{c}\text { Coordinates } \\
\text { RA, Dec (J2000) }\end{array}$ & $\begin{array}{c}\mathrm{H} \alpha \\
6562.8\end{array}$ & $\begin{array}{c}\mathrm{H} \beta \\
\lambda 4861.3\end{array}$ & $\begin{array}{c}{[\mathrm{O} \mathrm{III}]} \\
\lambda 5006.8\end{array}$ & $\begin{array}{c}{[\mathrm{O} \mathrm{III}]} \\
\lambda 4958.9\end{array}$ & $\begin{array}{c}{[\mathrm{O} \text { II }]} \\
\lambda 3727.0\end{array}$ & $\begin{array}{c}\mathrm{H} \gamma \\
\lambda 4340.5\end{array}$ & $\begin{array}{c}{[\mathrm{Ne} \text { III }]} \\
\lambda 3967.5\end{array}$ & $\begin{array}{c}{[\mathrm{N} \mathrm{II}]} \\
\lambda 6583.4\end{array}$ \\
\hline 1 & $15: 03: 52.65,-31: 39: 34.7$ & 7.4 & 1.9 & 6.4 & 1.7 & 10.6 & 0.6 & - & 2.4 \\
2 & $15: 03: 52.46,-31: 39: 34.3$ & 15 & 2.8 & 10.4 & 4.2 & 6.6 & 1.8 & 1.2 & - \\
3 & $15: 03: 52.45,-31: 39: 34.9$ & 34 & 6.2 & 28.2 & 5.8 & - & 1.9 & - & 3.8 \\
4 & $15: 03: 52.86,-31: 39: 33.3$ & 7.2 & 1.4 & 6.6 & 3.2 & - & - & - & 2.5 \\
94 & $15: 03: 52.07,-31: 39: 36.4$ & 3.7 & - & - & - & - & - & - & 0.2 \\
98 & $15: 03: 52.16,-31: 39: 36.0$ & 9.45 & 2.66 & 9.50 & 1.65 & - & - & 0.13 & 0.22 \\
113 & $15: 03: 52.54,-31: 39: 34.5$ & 10 & 2.5 & 11 & 4.0 & - & - & - & - \\
117 & $15: 03: 52.62,-31: 39: 34.2$ & 2.23 & 0.43 & 1.9 & 0.46 & - & - & - & - \\
136 & $15: 03: 52.97,-31: 39: 32.9$ & 7.23 & - & 1.77 & 0.4 & - & 1.67 & - & - \\
\hline
\end{tabular}

Notes. For each object the first line is the measured line flux in units of $10^{-17} \mathrm{erg} \mathrm{s}^{-1} \mathrm{~cm}^{-2}$. All lines lie at a common redshift of $z=0.102 \pm 0.001$. 
$(\mathrm{RA}, \mathrm{Dec}=15: 03: 52.73,-31: 39: 34.4)$ is only visible in the NIR, object \#x (RA, Dec = 15:03:52.66, -31:39:34.1) only in the optical bands and lies $1^{\prime \prime}$ north-west of \#y (given our astrometric $1 \sigma$ error of about $0 . ' 3$, the positions of both objects disagree by $\sim 2.5 \sigma$ ). We identify the NIR object close to object A as the NIR counterpart of object K (Fig. 1).

Object \#x also showed up in our spectroscopy runs. Our FORS2 spectra reveal bright emission lines at $z=0.102$ arising from several positions along the suspected tidal stream in the east-west direction (including galaxy $\mathrm{C}$ ). The strongest are labelled blobs 1 and 2 (covered by our first slit position) and blobs 3 and 4 (covered by our second slit position; Fig. 4). In addition, we find five more emission-line regions, labelled according to their $y$-coordinates in the optical spectrum (blobs 94 , $98,113,117$, and 136). Within the astrometric errors, we identify blobs 1 and 117 as two different emission-line regions belonging to the aforementioned object \#x, and blobs 2 and 3 we identify with object K (Fig. 3; Table 3). The presence of the $\mathrm{H} \alpha$ line in blobs 94, 98, and 136 points to a larger extension of the galaxy complex C compared to its optical appearance on the Gemini $i$-band image.

\subsubsection{Star formation rate: optical data}

We performed extinction correction of the observed line flux first by correcting for the reddening due to Galactic dust along the line of sight $\left(E(B-V)_{\mathrm{Gal}}=0.17 \mathrm{mag}\right.$; Schlegel et al. 1998), using the Milky Way extinction law and a ratio of total-to-selective extinction of $R_{V}=3.08$ (Pei 1992), and thereafter by assuming again a Milky Way extinction law for the star-forming region(s) in the GRB host galaxy. Thereby, we followed Domínguez et al. (2013) and used the observed flux ratio $F(\mathrm{H} \alpha) / F(\mathrm{H} \beta)$ (after correction for Galactic extinction), which was compared with the theoretically expected flux ratio ${ }^{6}$ to obtain $E(B-V)_{\text {host }}$ (Table 4). The host-extinction values in the emission-line blobs derived with this approach lie between 0.2 and $1.7 \mathrm{mag}$, with the highest values for those regions that are covering object K (blobs 2 and 3). Additional information could come from the WISE satellite (Wright et al. 2010). However, no object was detected inside the XRT error circle, and we can only obtain upper limits: $\mathrm{W} 1(3.4 \mu \mathrm{m})>20.0$, W2 $(4.6 \mu \mathrm{m})>20.2$, W3 $(12 \mu \mathrm{m})>17.8$, and $\mathrm{W} 4(22 \mu \mathrm{m})>15.4(\mathrm{AB} \text { magnitudes })^{7}$.

The extinction-corrected $\mathrm{H} \alpha$ luminosities of these blobs lie between 0.3 and $5.3 \times 10^{40} \mathrm{erg} \mathrm{s}^{-1}$, with the lowest value belonging to blob 117 and the highest to blob 3. For comparison, the latter corresponds to the combined radiative output of about 300 stars of spectral type 05.

Star formation rates were calculated using the expressions given in, e.g., Kennicutt (1998) and Savaglio et al. (2009):

$$
\begin{aligned}
& \operatorname{SFR}(\mathrm{H} \alpha)=4.39 \times 10^{-42} \frac{L(\mathrm{H} \alpha)_{\text {corr }}}{\mathrm{erg} \mathrm{s}^{-1}} M_{\odot} \mathrm{yr}^{-1}, \\
& \operatorname{SFR}([\mathrm{O} \mathrm{II}])=5.54 \times 10^{-42} \frac{L([\mathrm{O} \mathrm{II}])_{\text {corr }}}{\operatorname{erg~s^{-1}}} M_{\odot} \mathrm{yr}^{-1},
\end{aligned}
$$

where $L$ is the corresponding luminosity in the (redshifted) line, $L=4 \pi d_{L}^{2} F, d_{L}$ is the luminosity distance, $F$ the observed and extinction-corrected line flux. The SFRs that are derived with this approach are small, at most $0.2 M_{\odot} \mathrm{yr}^{-1}$ (in blob 3; Table 4).

\footnotetext{
6 The deduced corrections for the host galaxy are based on a theoretical value $F(\mathrm{H} \alpha) / F(\mathrm{H} \beta)=2.86$, which corresponds to a gas temperature of $10000 \mathrm{~K}$ and an electron density of $100 \mathrm{~cm}^{-3}$ (Osterbrock 1989).

7 For the Vega-AB conversion see http://wise2.ipac.caltech. edu/docs/release/prelim/expsup/sec4_3g.html
}

Table 4. Deduced host extinction and extinction-corrected SFR

\begin{tabular}{|c|c|c|c|c|}
\hline $\begin{array}{r}\# \\
(1)\end{array}$ & $\begin{array}{l}\frac{F(\mathrm{H} \alpha)}{F(\mathrm{H} \beta)} \\
(2)\end{array}$ & $\begin{array}{c}E(B-V)_{\text {host }} \\
\text { (3) }\end{array}$ & $\begin{array}{c}S F R(\mathrm{H} \alpha) \\
\quad(4)\end{array}$ & $\begin{array}{c}S F R([\mathrm{O} \mathrm{II}]) \\
(5)\end{array}$ \\
\hline 1 & 3.32 & 0.13 & 0.02 & 0.06 \\
\hline 2 & 4.56 & 0.40 & 0.10 & 0.14 \\
\hline 3 & 4.67 & 0.42 & 0.23 & - \\
\hline 4 & 4.38 & 0.36 & 0.04 & - \\
\hline 98 & 3.03 & 0.05 & 0.02 & - \\
\hline 113 & 3.41 & 0.15 & 0.03 & - \\
\hline 117 & 4.42 & 0.37 & 0.01 & - \\
\hline
\end{tabular}
$\left(M_{\odot} \mathrm{yr}^{-1}\right)$.

Notes. Column 1 follows Table 3; Col. 2 is the observed line flux ratio; Col. 3 is the deduced host reddening (mag), both after correcting for Galactic extinction (see text). Errors in the resulting host extinctions are $0.80 \times E(B-V)$ (Domínguez et al. 2013). Columns 4 and 5 give the extinction-corrected SFR based on Eqs. (1) and (2).

The large equivalent width of the $\mathrm{H} \alpha$ and the [O III] (5007) line in blob 3 (Fig. 4) suggests that the galaxy-complex $\mathrm{C}$ is a member of the class of extreme emission-line galaxies. These galaxies are rare among local low-mass galaxies $(<0.5 \%$ of galaxies in SDSS; Kniazev et al. 2004) and often show a disturbed morphology (Amorín et al. 2014).

\subsubsection{Star formation rate: radio data}

Radio-continuum observations are insensitive to extinction by cosmic dust and could reveal whether there is additional optically hidden star formation activity. Indeed, we detect a single, relatively bright radio source inside the XRT error circle slightly west of object A at coordinates RA, Dec $(J 2000)=15: 03: 52.26$, $-31: 39: 34.9$, with an integrated flux density at $5.5 \mathrm{GHz}$ of $F_{v}=$ $46 \pm 16 \mu \mathrm{Jy}$ (Fig. 5). The deconvolution of this radio image suggests that it is a point source. This source lies about $2^{\prime \prime}$ north-east of blob 98, which showed up through its emission-line spectrum (Figs. 2, 4). It is not detected at $9.0 \mathrm{GHz}, F_{v}<18 \mu \mathrm{Jy}(3 \sigma)$, constraining the spectral slope $\left(F_{v} \sim v^{-\alpha}\right)$ to be $\gtrsim 1.0$ (based on the $1 \sigma$ lower limit for the flux at $5.5 \mathrm{GHz}$ ).

We do not detect galaxy $\mathrm{C}$ (as defined by the ellipse drawn in Fig. 3) in the radio band, either at $5.5 \mathrm{GHz}\left(F_{v}<16 \mu \mathrm{Jy}\right.$, $3 \sigma$; Fig. 5$)$ or at $9.0 \mathrm{GHz}\left(F_{v}<18 \mu \mathrm{Jy}, 3 \sigma\right)$. Following the procedure described in Bell (2003) and Michałowski et al. (2009), this translates into an upper limit for the SFR of object $\mathrm{C}$ of $1 M_{\odot} \mathrm{yr}^{-1}$. This agrees with the SFR derived from the optical emission-line data, which suggest a total extinction-corrected SFR in this galaxy of $\lesssim 1 M_{\odot} \mathrm{yr}^{-1}$ (Table 4). This situation is similar in regards to blobs 2 and 3 (or, equivalently, object K; Figs. 1, 2): we do not detect them in the radio band. In other words, there is not much optically hidden star formation taking place.

\subsection{What is the nature of the radio source inside the XRT error circle?}

At $5.5 \mathrm{GHz}$ we measure an offset of 1 ." 4 between the optical position of $\mathrm{A}$ and the radio source inside the XRT error circle. Given our synthesized beam size $(F W H M=4$ ". 2 along the major axis; Sect. 2.3) and signal-to-noise ratio (S/N) (peak flux divided by the rms of the background flux), the positional radio uncertainty is $0.6 F W H M /(S / N)=0 \prime^{\prime} 9$. The offset we measure is 

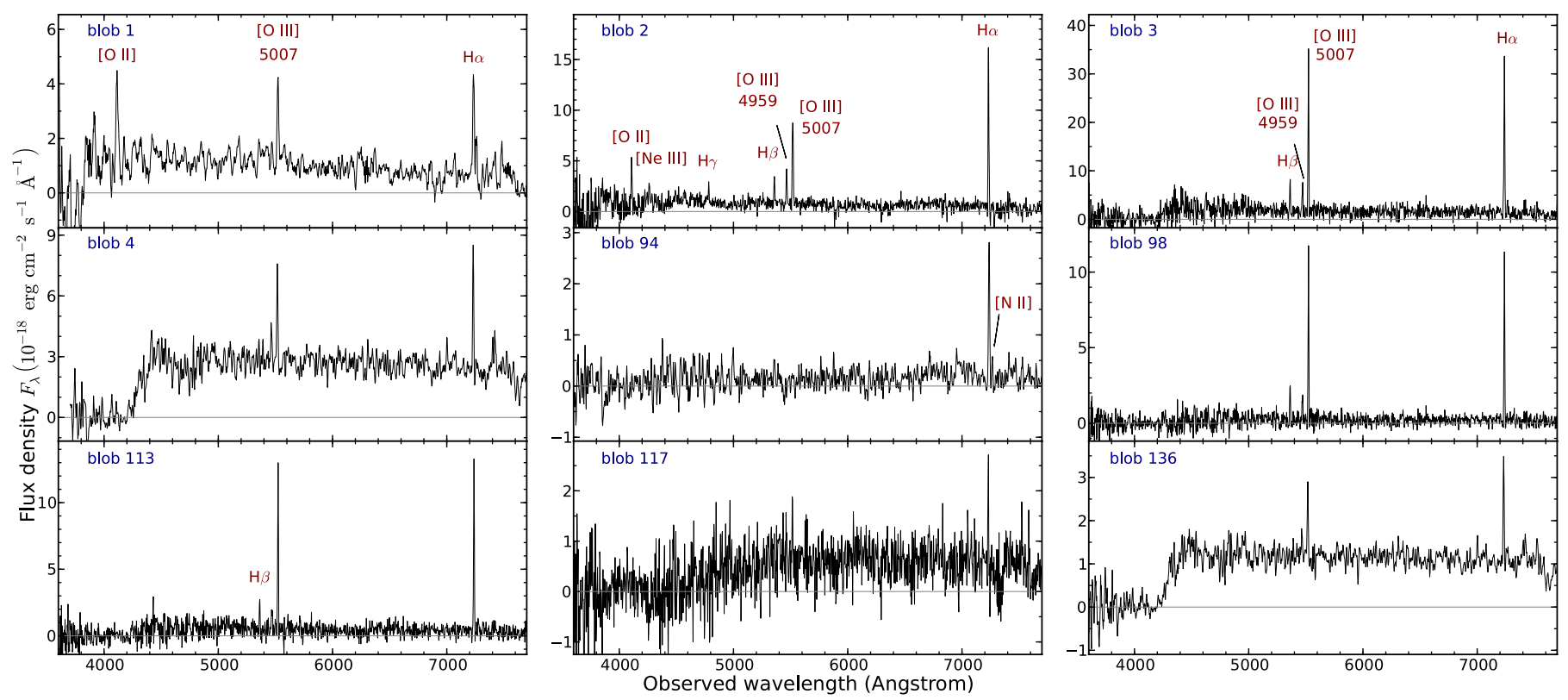

Fig. 4. Spectra of the nine blobs we found in our VLT/FORS2 2D spectra that show various emission lines, indicative of star formation at a common redshift of $z=0.102$ ( $x$ axes are observed wavelengths). Blobs 94 and 98 have no optical counterpart on the Gemini $i$-band image but showed up in the trace of the FORS2 spectrum. In blobs 2 and 3 the $\mathrm{H} \alpha$ and the [O III] (5007) line are very strong. In blob 3 we measure an equivalent width of $220 \pm 20 \AA$ and $170 \pm 20 \AA$, respectively.

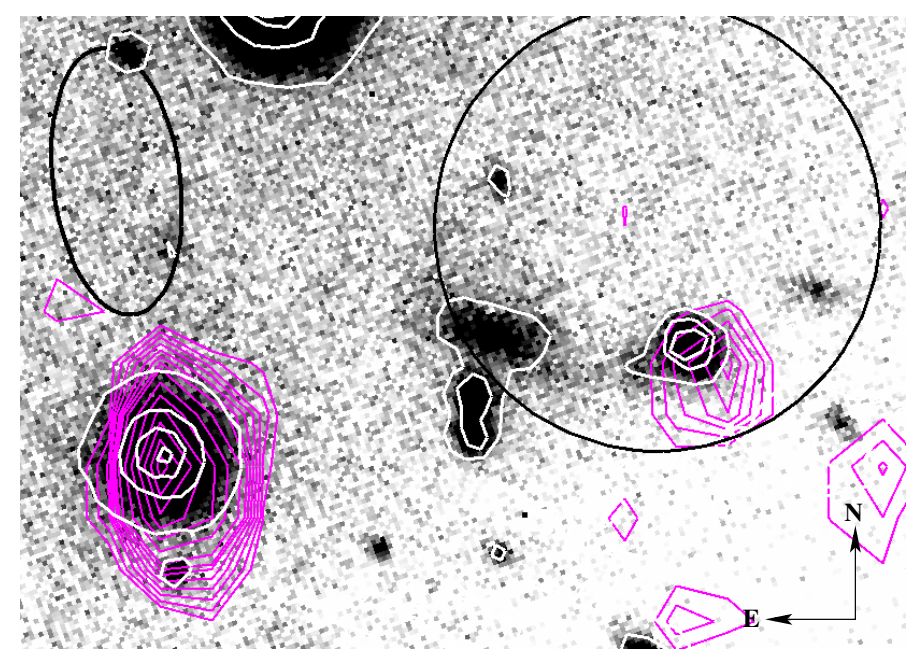

Fig. 5. The field of GRB 100628A as seen by ATCA in the $5.5 \mathrm{GHz}$ band overlaid in Fig. 1. Radio contour levels (in magenta) run from $F_{v}=10$ to $25 \mu \mathrm{Jy}$ in steps of $2.5 \mu \mathrm{Jy}$ and then from 30 to $100 \mu \mathrm{Jy}$ in steps of $10 \mu \mathrm{Jy}$. The size of the synthesized beam is 2 .' $2 \times 4$ ". 0 (upper left corner). Inside the XRT error circle, we do not detect object $\mathrm{C}$, but we detect emission close to object A (see text for details). In addition, we detect object $\mathrm{D}$ as a bright point source. Overlaid are the optical contours (white).

therefore not statistically significant $(<3 \sigma)$. This leaves us with three possibilities to explain the nature of this radio source.

(1) The source of the radio flux is indeed object A, i.e., the $\mathrm{K}$ star. In fact, the main sequence stars of spectral type $\mathrm{K}$ are known to be possible radio emitters (e.g., Güdel 1992, 2002). However, in this case it seems to be very unlikely because the extinction-corrected optical magnitude of this star implies a distance of a few $\mathrm{kpc}$, which then leads to a radio luminosity notably exceeding the observed radio luminosities of Galactic
K stars (Güdel 1992). (2) The source of the radio emission is star formation in the tidal tail of galaxy $\mathrm{C}$. In that case, the flux density should scale as $F_{v} \sim v^{-\alpha}$, with a canonical value of $\alpha=0.75$ (but an observed range between about 0.5 and 1; e.g., Condon 1992; Ibar et al. 2010). Our radio data do not exclude this kind of a scenario (Sect. 3.1.2). Interpreting the flux at $5.5 \mathrm{GHz}$ with this approach, and following Michałowski et al. (2009), this then translates into an unobscured SFR of $2 \pm 0.5 M_{\odot} \mathrm{yr}^{-1}$. (3) The radio source is the three-year-old radio afterglow of GRB 100628A. This source would then be as bright as, e.g., the twoyear-old radio afterglow of GRB 100418A (Moin et al. 2013), a long burst that lies at $z=0.624$ (Antonelli et al. 2010; Cucchiara $\&$ Fox 2010). If this radio source lies at $z=0.102$, then its radio spectral luminosity at $5.5 \mathrm{GHz}$ is about $1 \times 10^{28} \mathrm{erg} \mathrm{s}^{-1} \mathrm{~Hz}^{-1}$. Compared with the present world sample of radio observations of long-GRB afterglows this is a rather modest luminosity for a three-year-old event (see Chandra \& Frail 2012, their Fig. 6). Nevertheless, this could be an unlikely case for a short burst, which would usually be expected to explode in low-density environments. However, we note that our VLT spectroscopy revealed ongoing star formation activity in that direction. In other words, if GRB 100628A came from that region (at $z=0.102$ ), there could be a high enough interstellar gas density to feed its radio afterglow (and to dim its optical afterglow). Future second-epoch observations could reveal whether this radio source is fading or not. Unfortunately, these observations might have to wait until the year 2016+ before any possible fading could be established or ruled out.

\subsection{Outside the XRT error circle: Galaxy D}

According to our present understanding, the progenitors of short bursts could be several Gyr old when they merge (see e.g., Berger 2014, for a review). The offset distribution between a short GRB and its host (if known) could provide critical 


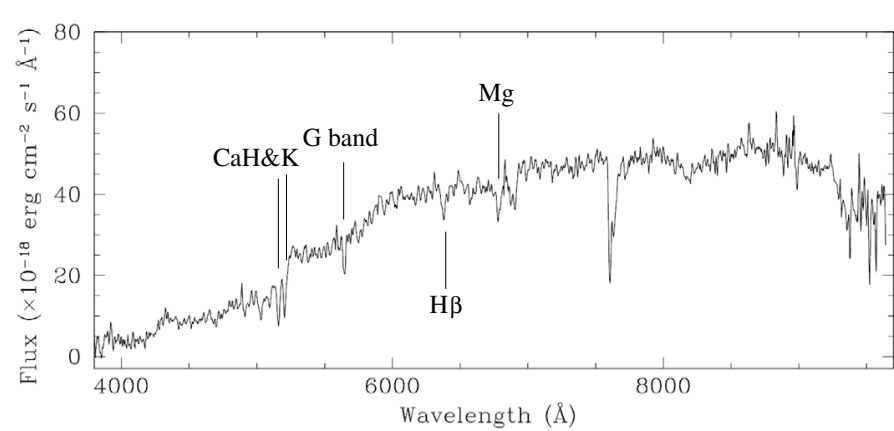

Fig. 6. VLT/FORS2 spectrum of object D reveals that this is an elliptical galaxy at a redshift of $z=0.311$. The strong absorption at $7600 \AA$ is due to a telluric band ( $A$ band).

constraints on progenitor models. However, the offset between the afterglow location and the host centre is a complex convolution of the progenitor birth position within the host, the delay time distribution resulting from the properties of the binary system, the multiple systemic kicks generated during the formation of the progenitor pair, the gravitational potential of the host, and the orientation of the host in relation to the initial kick velocity vector (e.g., Fryer et al. 1999; Bloom et al. 1999; Belczynski et al. 2006; Behroozi et al. 2014). Even for massive host galaxies like the Milky Way or M31, a favourably oriented kick with magnitude of a few hundred $\mathrm{km} \mathrm{s}^{-1}$, in combination with a Gyr delay time, can cause the merger to take place in the realm of the outer halo. For less massive hosts these conditions would ensure that the progenitor system escapes the host potential altogether, subsequently resulting in a merger and its associated GRB in intergalactic space. In fact, several cases of apparently hostless short GRBs are known (e.g., Tunnicliffe et al. 2014), perhaps supporting this scenario. This possibility points our attention to object D (Fig. 1).

Object D lies 17" away from the centre of the (90\% c.l.) XRT error circle. This galaxy has observed AB magnitudes of $g^{\prime} r^{\prime} i^{\prime} z^{\prime} J H K_{\mathrm{S}}=22.46 \pm 0.07,20.72 \pm 0.02,20.17 \pm 0.02,19.84 \pm$ $0.02,18.69 \pm 0.02,18.35 \pm 0.03$, and $18.06 \pm 0.05 \mathrm{mag}$. In our ATCA radio-continuum observations it stands out as a bright, point-like source with an integrated flux density of $F_{v}(5.5 \mathrm{GHz})$ $=151 \pm 23 \mu \mathrm{Jy}$ and $F_{v}(9.0 \mathrm{GHz})=83 \pm 28 \mu \mathrm{Jy}$ (Fig. 5). In the entire Swift/BAT error circle (radius 2.1 arcmin, 90\% c.l.; Immler et al. 2010) it is the second-brightest radio source. In combination with our VLT/FORS2 spectroscopy, this identifies $\mathrm{D}$ as a radio-bright elliptical galaxy at a redshift of $z=0.311$ (Fig. 6) ${ }^{8}$.

The galaxy is also detected in the WISE satellite W1 and $\mathrm{W} 2$ bands with $\mathrm{W} 1(3.4 \mu \mathrm{m})=18.65 \pm 0.08$ and $\mathrm{W} 2(4.6 \mu \mathrm{m})=$ $18.87 \pm 0.18$, while it is not detected at longer wavelengths: $\mathrm{W} 3(12 \mu \mathrm{m})>17.8$, and $\mathrm{W} 4(22 \mu \mathrm{m})>15.4$ (AB magnitudes). In the WISE colour-colour diagram (Wright et al. 2010) it lies in the region occupied by active galactic nuclei that are ellipticals with a pure absorption line spectrum. As such, it also roughly follows the $K-z$ (Vega $K$-band magnitude-redshift) relation for radio galaxies and its approximated radiation power $P$ at $20 \mathrm{GHz}$ (based on the observed spectral slope between 5.5 and $9.0 \mathrm{GHz}$ ) of $\log P \sim 29.0 \pm 0.3\left(\mathrm{erg} \mathrm{s}^{-1} \mathrm{~Hz}^{-1}\right)$ puts it at the lower end of the radio luminosity function of local radio galaxies (Sadler et al. 1999, 2014).

\footnotetext{
8 At this redshift the luminosity distance is $4.96 \times 10^{27} \mathrm{~cm}, m-M=$ $41.03 \mathrm{mag}$, the look-back time is $3.49 \mathrm{Gyr}$, and $1^{\prime \prime}$ corresponds to $4.54 \mathrm{kpc}$.
}

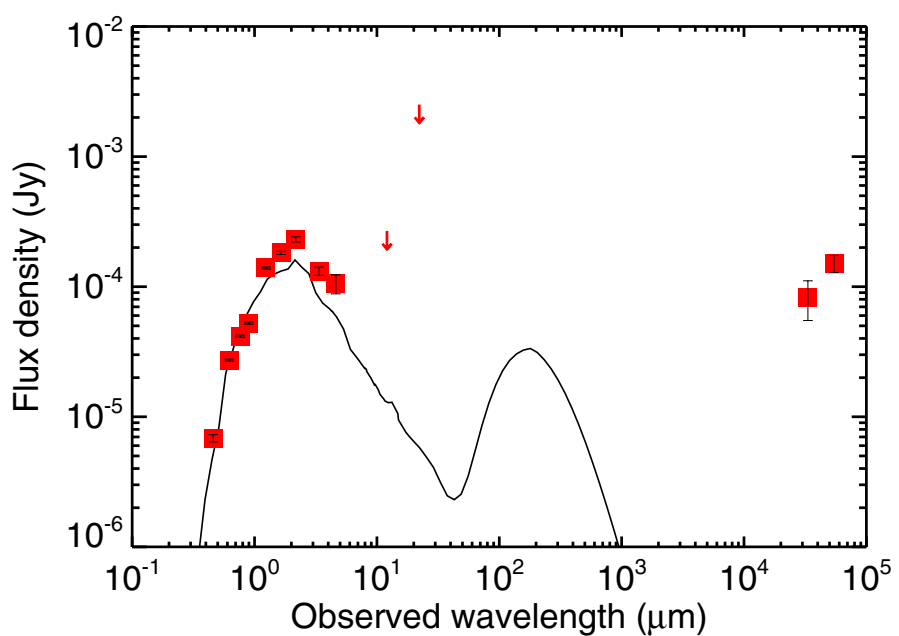

Fig. 7. GRASIL fit of the (Galactic extinction-corrected) SED of object D from the GROND $g^{\prime}$ to the WISE W4 band using the template of an elliptical galaxy. The radio fluxes have not been used in this fit since they are related to AGN activity.

The $K_{\mathrm{s}}$-band luminosity of galaxy $\mathrm{D}$ is very high, $\log \left(L / L_{\odot}\right)=11.24$, but not unusual for this class of elliptical galaxies (Sadler et al. 2014). A GRASIL (Silva et al. 1998) fit of the broadband SED (Michałowski et al. 2008, 2010, 2012, 2014) from the optical to the WISE bands provides a stellar mass of $M_{\star}=7.7 \times 10^{10} M_{\odot}($ Salpeter IMF), no evidence for star formation activity (current $S F R=0 M_{\odot} \mathrm{yr}^{-1}$ ), and a metallicity of $\log Z / Z_{\odot}=0.02$ (Fig. 7)

The global properties of this galaxy are nearly identical to that of the likely host of the first well-localized short burst, GRB 050509B, a giant E1 elliptical galaxy at a redshift of $z=0.225$ (Gehrels et al. 2005; Bloom et al. 2006). Also for this burst no optical afterglow was detected and the angular offset between the elliptical galaxy and the centre of the UVOT-enhanced $r=3$.' 6 XRT error circle is in the order of $10^{\prime \prime}$. According to Kochanek et al. (2001), as already outlined by Gehrels et al. (2005), these luminous early-type galaxies are quite rare; the comoving number density of early-type galaxies as luminous as object $\mathrm{D}$ with $M_{K_{\mathrm{s}}} \sim-24.75 \mathrm{mag}$ (Vega) is $\sim 3 \times 10^{-4}(\mathrm{Mpc} / \mathrm{h})^{-3} \mathrm{mag}^{-1}$, where $h$ is the Hubble constant in units of $100 \mathrm{~km} \mathrm{~s}^{-1} \mathrm{Mpc}^{-1}$. As discussed by Gehrels et al. (2005) and Bloom et al. (2006), these galaxies are ideal birthplaces for compact stellar binaries, i.e., short-GRB progenitors. Adopting again the methodology used in Sect. 3.1, but now using number counts of E/S0 galaxies in the $I$ (Vega) band (Huang et al. 1998), the probability $p$ of finding this kind of galaxy with $I$ (Vega) $\leq 19.5$ (after applying a AB-Vega conversion of $\sim 0.4 \mathrm{mag}$ and after correcting for the Galactic extinction along the line of sight, $0.3 \mathrm{mag}$ in the GROND $i^{\prime}$ band) inside a circle on the sky with a radius of $17^{\prime \prime}$ is about $p=5 \%$. In other words, galaxy D is clearly another host-galaxy candidate.

Is it reasonable to assume that the elliptical galaxy could be the GRB host? Its minimum distance to those of the outer edge of the error circle is $10^{\prime \prime}$. At a redshift of $z=0.311$ this corresponds to a projected distance of $45 \mathrm{kpc}$. While this would be an unusually large distance for a long-GRB progenitor (e.g., Bloom et al. 2002), for a short-GRB progenitor this is not the case. On the other hand, we caution that from the observational side the offset distribution of hostless short-burst afterglows (Tunnicliffe et al. 2014) from their host galaxies is unknown. 


\subsection{Pro and contra $z=0.102$ for $G R B 100628 A$}

\subsubsection{Host galaxy properties}

The current world sample of short-GRB host galaxies is still very small, only $\sim 30$ are known. The majority $(\sim 3 / 4)$ of the so far well-studied short-GRB hosts are star-forming spiral and irregular galaxies, while only a minority $(\sim 1 / 4)$ are ellipticals (for a review, see Berger 2014, and references therein). Besides, there has not been any obvious observed trend between the morphology of the host galaxies and their redshift. Short-GRB host galaxy masses, star formation rates, and optical luminosities span over a wide range of values. Neither the potential spiral/interacting galaxy at $z=0.102$ nor the giant elliptical at $z=0.311$ would be outliers in this respect.

Other physical parameters have to be considered to evaluate which redshift solution fits best into the present world sample of short bursts.

\subsubsection{Short-GRB redshift distribution}

If galaxy C is the host of GRB 100628A then, after GRB 061201 (Stratta et al. 2007) and GRB 080905A (Rowlinson et al. 2010), this would be the third case of a short-GRB host lying at a redshift $z \sim 0.1$. How likely is this kind of scenario?

Several authors have calculated the expected redshift distribution of short-duration GRBs (e.g., Guetta \& Piran 2006; Behroozi et al. 2014). Following Behroozi et al. (2014), the observed rate (per unit time) as a function of $z$ is (their Eq. (3))

$N(<z)_{\text {obs }}=\int_{0}^{\infty} N(z)_{\text {intrin }} \frac{\epsilon_{\text {detector }}}{1+z} \frac{\mathrm{d} V}{\mathrm{~d} z} \mathrm{~d} z$

where $\mathrm{d} V / \mathrm{d} z$ is the comoving volume per redshift bin (e.g., Wang $\&$ Dai 2009), $(1+z)$ the time dilution factor, and the function $\epsilon_{\text {detector }}$ takes the mean instrumental detection probability for short GRBs at redshift $z$ into account. According to Behroozi et al. (2014), in the case of Swift this function is best described by $\epsilon_{\text {detector }} \propto \exp (-4.3 z)$.

We used the models plotted in Fig. 1 in Behroozi et al. (2014) as a numerical input to calculate $N(<z)_{\text {obs }}$. These models refer to three different analytical forms of the assumed delay time distributions of merging compact stellar binary systems. In all of these cases, we find that among the 24 Swift-detected short GRBs with a precise spectroscopic redshift (by mid-2013; Table 1 in Berger 2014, excluding the potential long burst 090426A), at most one event should lie at $z<0.12$, while two are observed (GRB 061201 at $z=0.111$ and GRB 080905A at $z=0.122$ ). This seems to disfavour the idea that another third event came from such a low redshift.

However, some caution is necessary here. Firstly, contrary to the case of GRB 080905A, the redshift of GRB 061201 is not securely known. Even though the burst had a well-detected optical afterglow, i.e., sub-arcsec localization, no underlying host galaxy was found and the redshift refers to the most probable host, which was identified based on its apparent magnitude, and lies 17 arcsec away (Stratta et al. 2007). It is therefore well possible that this is not the GRB host galaxy. Secondly, the observational uncertainties in the assumed delay time distributions are basically unknown. It would be much better to find strong physical arguments that can rule out a redshift of $z=0.102$ for GRB 100628A. however, we do not have such arguments at hand.

\subsubsection{Prompt and afterglow statistics}

Following the procedure described in Bloom et al. (2001), as it has been implemented in Kann et al. (2010, 2011), and using the spectral and energy parameters as given by Barthelmy et al. (2010), the bolometric isotropic equivalent energy release of the burst was $\log E_{\text {iso,bol }}(\mathrm{erg})=48.07_{-0.07}^{+0.13}$, and $49.05_{-0.07}^{+0.13}$ for $z=$ 0.102 and $z=0.311$, respectively. For a supposed redshift of $z=0.102$, this would rank GRB 100628A as the least energetic short burst recorded to date (closely followed by GRB 050509B with $\log E_{\text {iso,bol }}(\mathrm{erg})=48.38_{-0.23}^{+0.45}$, while for $z=0.311$ the burst would fall into the region occupied by other known low-energy short GRBs (see Kann et al. 2011, their Table 3).

To better determine the most likely redshift of GRB 100628 A, i.e., $z=0.102$ or 0.311 , we checked several empirical correlations between various physical parameters during the prompt emission phase and the afterglow phase. Recently, D'Avanzo et al. (2012) and D'Avanzo et al. (2014) explored the correlations between X-ray afterglow luminosity, $L_{X}$ at various host-frame times after the burst on the one hand and the GRB peak energy in the host frame, $E_{\text {peak }}$, the isotropic energy release, $E_{\text {iso,bol }}$ as well as the isotropic peak luminosity during the prompt phase, $L_{\text {iso }}$, on the other hand. We focussed on their 11-hr sample since for both possible redshifts of interest it includes the host-frame time when the first X-ray photons of the afterglow of GRB 100628A were detected (Evans et al. 2007, 2009) ${ }^{9}$. Given that the X-ray afterglow was very faint and its light curve is not well determined, the outcome of this study has to be taken with care.

We find that based on this comparison neither of the two redshifts is really excluded ${ }^{10}$. In particular, both cases match the observed $L_{\mathrm{X}}-E_{\text {iso,bol }}$ relation for short GRBs (see also Fig. 14 in Berger 2014), even though the higher redshift $(z=0.311)$ is slightly preferred. Moreover, for both redshifts the burst does not

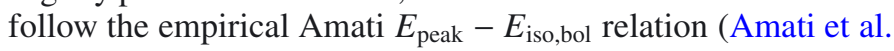
2008), as is typical for short GRBs (Amati et al. 2007).

Finally, the X-ray afterglow luminosity alone does not rule out any of the two redshifts. Both luminosities fit well into the statistics of X-ray afterglow luminosities of short bursts summarized in Nicuesa Guelbenzu et al. (2012). Even though, the higher redshift would fit slightly better than the lower one again.

\section{Summary}

Based on a combination of deep multi-colour imaging in the optical/NIR bands (GROND, Gemini), optical spectroscopy (VLT/FORS2 and X-Shooter) and deep ATCA radio-continuum observations, we have studied two host-galaxy candidates for the short GRB 100628A.

Inside its $r=7^{\prime \prime}$ (90\% c.1.) X-ray error circle lies an interacting, star-forming galaxy system at a redshift of $z=0.102$. Its relatively bright apparent magnitude as well as peculiar morphology classifies this galaxy system as the best host-galaxy candidate among all (otherwise much fainter) galaxies visible inside the error circle. If galaxy $\mathrm{C}$ is the host, it would be the nearest host galaxy of a short burst known thus far, followed in redshift space by GRB 080905A at $z=0.1218$ (Rowlinson et al. 2010). The galaxy itself is not detected in the radio band, but we have found a radio source inside the error circle that could be related

\footnotetext{
9 For the data, see http://www.swift.ac.uk/xrt_live_cat/

${ }^{10}$ For $z=0.102$, we have $\log E_{\text {peak }}(\mathrm{keV})=1.9, \log L_{\text {iso }}\left(\mathrm{erg} \mathrm{s}^{-1}\right)=$ $49.2, \log L_{\mathrm{X}}\left(\mathrm{erg} \mathrm{s}^{-1}\right)=42.1, t_{\text {host }}=8.2 \mathrm{~h}$. For $z=0.311$, it is $\log$ $E_{\text {peak }}(\mathrm{keV})=2.0, \log L_{\text {iso }}\left(\operatorname{erg~s}^{-1}\right)=50.3, \log L_{\mathrm{X}}\left(\mathrm{erg} \mathrm{s}^{-1}\right)=43.2$ at $t_{\text {host }}=6.9 \mathrm{~h}$.
} 
to the star-forming activity in its extended tidal stream, though we cannot exclude at this time that this is the fading radio afterglow. If the progenitor of GRB 100628A was formed as a result of the observed star formation episode in this galaxy, it could have been a member of a young stellar population.

Interestingly, slightly outside the X-ray error circle, 17 " away from its centre, our multi-band data revealed a second host-galaxy candidate, an elliptical galaxy at a redshift of $z=$ 0.311 . It stands out by its radio brightness (observed flux density) and its $K$-band luminosity. The latter, $L_{K \mathrm{~s}} \sim 2 \times 10^{11} L_{\odot}$, is as large as the one of the luminous elliptical host galaxy of the first well-localized short burst, GRB 050509B at $z=0.225$. If GRB 100628A originated from this galaxy, then its progenitor would have been a member of an old stellar population and its projected offset from its host at the time of its explosion would have been $\sim 45 \mathrm{kpc}$.

Unfortunately, no strong arguments can be offered that clearly prefer one host-galaxy candidate over the other, i.e., that would allow us to decide between the spiral/interacting galaxy at $z=0.102$ and the giant elliptical at $z=0.311$. None of the discussed properties rule out any redshift solution, including the host galaxy properties, the physical parameters that describe the prompt phase and characterize the X-ray afterglow, as well as the different empirical correlations between them. The higher redshift solution, however, fits slightly better into the ensemble properties of short GRBs known thus far.

Our results show once more that even for short GRBs additional radio observations can shed new light on the nature of their suspected host galaxies. In the case of the host of the short burst GRB 071227 they revealed its star-bursting nature (Nicuesa Guelbenzu et al. 2014), in the case discussed here they could indicate a second host-galaxy candidate. Obviously, radio data helps to gain a more complete view of the nature of the galaxies that hosted short GRBs.

Acknowledgements. A.N.G. acknowledges support by M. Guelbenzu and by grant DFG Kl 766/16-1. A.N.G. and S. Schmidl acknowledge support by the Thüringer Ministerium für Bildung, Wissenschaft und Kultur under FKZ 12010514. A.N.G. thanks Helmut Meusinger, Thüringer Landessternwarte, for delightful discussions. M.J.M acknowledges the support of the Science and Technology Facilities Council. D.A.K. acknowledges support by TLS Tautenburg, MPE Garching and DFG Kl/766 16-3. A.R. acknowledges support by TLS Tautenburg and DFG Kl/766 13-2. L.K.H., E.P, and A.R. are grateful for support from PRIN-INAF 2012/13. S. Schulze acknowledges support from CONICYTChile FONDECYT 3140534, Basal-CATA PFB-06/2007, and Project IC120009 "Millennium Institute of Astrophysics (MAS)" of Iniciativa Científica Milenio del Ministerio de Economía, Fomento y Turismo. Part of the funding for GROND (both hardware and personnel) was generously granted by the LeibnizPrize to G. Hasinger (DFG grant HA 1850/28-1). The Australia Telescope is funded by the Commonwealth of Australia for operation as a National Facility managed by CSIRO. This publication makes use of data products from the Widefield Infrared Survey Explorer, which is a joint project of the University of California, Los Angeles, and the Jet Propulsion Laboratory/California Institute of Technology, funded by the National Aeronautics and Space Administration. A.N.G. and S.K. thank Catarina Ubach \& Sarah Maddison, Swinburne University, Ivy Wong, CSIRO Sydney, and Jamie Stevens, CSIRO Narrabri, for helpful discussions and observing guidance. This work made use of data supplied by the UK Swift Science Data Centre at the University of Leicester. We thank the anonymous referee for very valuable comments.

\section{References}

Amati, L., Della Valle, M., Frontera, F., et al. 2007, A\&A, 463, 913 Amati, L., Guidorzi, C., Frontera, F., et al. 2008, MNRAS, 391, 577 Amorín, R., Sommariva, V., Castellano, M., et al. 2014, A\&A, 568, L8 Antonelli, L. A., Maund, J. R., Palazzi, E., et al. 2010, GRB Coordinates Network, 10620, 1

Barthelmy, S. D., Barbier, L. M., Cummings, J. R., et al. 2005, Space Sci. Rev., 120,143
Barthelmy, S. D., Sakamoto, T., Baumgartner, W. H., et al. 2010, GRB Coordinates Network, 10896, 1

Beckmann, V., Beck, M., Ferrigno, C., et al. 2010, GRB Coordinates Network, 10898, 1

Behroozi, P. S., Ramirez-Ruiz, E., \& Fryer, C. L. 2014, ApJ, 792, 123

Belczynski, K., Perna, R., Bulik, T., et al. 2006, ApJ, 648, 1110

Bell, E. F. 2003, ApJ, 586, 794

Berger, E. 2010a, GRB Coordinates Network, 10942, 1

Berger, E. 2010b, GRB Coordinates Network, 10943, 1

Berger, E. 2014, ARA\&A, 52, 43

Berger, E., Guver, T., \& Fong, W. 2010a, GRB Coordinates Network, 10911, 1

Berger, E., Guver, T., Fong, W., \& Chornock, R. 2010b, GRB Coordinates Network, 10902, 1

Berger, E., Zauderer, B. A., Levan, A., et al. 2013, ApJ, 765, 121

Bloom, J. S., Sigurdsson, S., \& Pols, O. R. 1999, MNRAS, 305, 763

Bloom, J. S., Frail, D. A., \& Sari, R. 2001, AJ, 121, 2879

Bloom, J. S., Kulkarni, S. R., \& Djorgovski, S. G. 2002, AJ, 123, 1111

Bloom, J. S., Prochaska, J. X., Pooley, D., et al. 2006, ApJ, 638, 354

Burenin, R., Khorunzhev, G., Sazonov, S., et al. 2010, GRB Coordinates Network, 10900, 1

Burrows, D. N., Hill, J. E., Nousek, J. A., et al. 2005, Space Sci. Rev., 120, 165 Cenko, S. B., Perley, D. A., Bloom, J. S., Morgan, A. N., \& Cucchiara, A. 2010, GRB Coordinates Network, 10946, 1

Chandra, P., \& Frail, D. A. 2012, ApJ, 746, 156

Condon, J. J. 1992, ARA\&A, 30, 575

Cucchiara, A., \& Fox, D. B. 2010, GRB Coordinates Network, 10624, 1

D'Avanzo, P., Salvaterra, R., Sbarufatti, B., et al. 2012, MNRAS, 425, 506

D’Avanzo, P., Salvaterra, R., Bernardini, M. G., et al. 2014, MNRAS, 442, 2342

Domínguez, A., Siana, B., Henry, A. L., et al. 2013, ApJ, 763, 145

Evans, P. A., Beardmore, A. P., Page, K. L., et al. 2007, A\&A, 469, 379

Evans, P. A., Beardmore, A. P., Page, K. L., et al. 2009, MNRAS, 397, 1177

Fryer, C. L., Woosley, S. E., \& Hartmann, D. H. 1999, ApJ, 526, 152

Gehrels, N., Sarazin, C. L., O’Brien, P. T., et al. 2005, Nature, 437, 851

Goldoni, P., Royer, F., François, P., et al. 2006, in SPIE Conf. Ser., 6269

Greiner, J., Bornemann, W., Clemens, C., et al. 2008, PASP, 120, 405

Güdel, M. 1992, A\&A, 264, L31

Güdel, M. 2002, ARA\&A, 40, 217

Guetta, D., \& Piran, T. 2006, A\&A, 453, 823

Henriques, B. M. B., White, S. D. M., Lemson, G., et al. 2012, MNRAS, 421, 2904

Hjorth, J., \& Bloom, J. S. 2012, The Gamma-Ray Burst - Supernova Connection (Cambridge: Cambridge University Press), 169

Horne, K. 1986, PASP, 98, 609

Huang, J.-S., Cowie, L. L., \& Luppino, G. A. 1998, ApJ, 496, 31

Ibar, E., Ivison, R. J., Best, P. N., et al. 2010, MNRAS, 401, L53

Immler, S. 2010, GRB Coordinates Network, 10901, 1

Immler, S., Starling, R. L. C., Evans, P. A., Barthelmy, S. D., \& Sakamoto, T. 2010, GCN Rep., 290, 1

Kann, D. A., Klose, S., Zhang, B., et al. 2010, ApJ, 720, 1513

Kann, D. A., Klose, S., Zhang, B., et al. 2011, ApJ, 734, 96

Kennicutt, Jr., R. C. 1998, ARA\&A, 36, 189

Kniazev, A. Y., Pustilnik, S. A., Grebel, E. K., Lee, H., \& Pramskij, A. G. 2004, ApJS, 153, 429

Kochanek, C. S., Pahre, M. A., Falco, E. E., et al. 2001, ApJ, 560, 566

Kouveliotou, C., Meegan, C. A., Fishman, G. J., et al. 1993, ApJ, 413, L101

Krühler, T., Küpcü Yoldaş, A., Greiner, J., et al. 2008, ApJ, 685, 376

Küpcü Yoldaş, A., Krühler, T., Greiner, J., et al. 2008, in AIP Conf. Ser. 1000, eds. M. Galassi, D. Palmer, \& E. Fenimore, 227

Lee, W. H., \& Ramirez-Ruiz, E. 2007, New J. Phys., 9, 17

Leibler, C. N., \& Berger, E. 2010, ApJ, 725, 1202

Levan, A. J., Tanvir, N. R., Wiersema, K., \& Cenko, S. B. 2010, GRB Coordinates Network, 10909, 1

Mazets, E. P., \& Golenetskii, S. V. 1981, Ap\&SS, 75, 47

Michałowski, M. J., Hjorth, J., Castro Cerón, J. M., \& Watson, D. 2008, ApJ, 672,817

Michałowski, M. J., Hjorth, J., Malesani, D., et al. 2009, ApJ, 693, 347

Michałowski, M., Hjorth, J., \& Watson, D. 2010, A\&A, 514, A67

Michałowski, M. J., Dunlop, J. S., Cirasuolo, M., et al. 2012, A\&A, 541, A85

Michałowski, M. J., Hayward, C. C., Dunlop, J. S., et al. 2014, A\&A, 571, A75

Moin, A., Chandra, P., Miller-Jones, J. C. A., et al. 2013, ApJ, 779, 105

Nakar, E. 2007, Phys. Rep., 442, 166

Nicuesa Guelbenzu, A., Klose, S., Greiner, J., et al. 2012, A\&A, 548, A101

Nicuesa Guelbenzu, A., Klose, S., Michalowski, M. J., et al. 2014, ApJ, 789, 45

Norris, J. P., Cline, T. L., Desai, U. D., \& Teegarden, B. J. 1984, Nature, 308, 434

Osterbrock, D. E. 1989, in Astrophysics of gaseous nebulae and active galactic nuclei (University Science Books), 422 
Pei, Y. C. 1992, ApJ, 395, 130

Perley, D. A., Modjaz, M., Morgan, A. N., et al. 2012, ApJ, 758, 122

Roming, P. W. A., Kennedy, T. E., Mason, K. O., et al. 2005, Space Sci. Rev., 120,95

Rowlinson, A., Wiersema, K., Levan, A. J., et al. 2010, MNRAS, 408, 383

Sadler, E. M., McIntyre, V. J., Jackson, C. A., \& Cannon, R. D. 1999, PASA, 16, 247

Sadler, E. M., Ekers, R. D., Mahony, E. K., Mauch, T., \& Murphy, T. 2014 MNRAS, 438, 796

Savaglio, S., Glazebrook, K., \& Le Borgne, D. 2009, ApJ, 691, 182

Schlegel, D. J., Finkbeiner, D. P., \& Davis, M. 1998, ApJ, 500, 525

Silva, L., Granato, G. L., Bressan, A., \& Danese, L. 1998, ApJ, 509, 103

Skrutskie, M. F., Cutri, R. M., Stiening, R., et al. 2006, AJ, 131, 1163

Spergel, D. N., Verde, L., Peiris, H. V., et al. 2003, ApJS, 148, 175

Starling, R. L. C., \& Immler, S. 2010, GRB Coordinates Network, 10899, 1
Starling, R. L. C., Beardmore, A. P., \& Immler, S. 2010a, GRB Coordinates Network, 10907, 1

Starling, R. L. C., Evans, P. A., \& Immler, S. 2010b, GRB Coordinates Network, 10941, 1

Stratta, G., D’Avanzo, P., Piranomonte, S., et al. 2007, A\&A, 474, 827

Suzuki, D., Omori, K., Hayashi, F., et al. 2010, GRB Coordinates Network, 10904, 1

Tody, D. 1993, ASP Conf. Ser., 52, 173

Tunnicliffe, R. L., Levan, A. J., Tanvir, N. R., et al. 2014, MNRAS, 437, 1495

Updike, A., Nardini, M., Afonso, P., et al. 2010, GRB Coordinates Network, 10910, 1

Wang, F. Y., \& Dai, Z. G. 2009, MNRAS, 400, L10

Wilson, W. E., Ferris, R. H., Axtens, P., et al. 2011, MNRAS, 416, 832

Woosley, S. E., \& Bloom, J. S. 2006, ARA\&A, 44, 507

Wright, E. L., Eisenhardt, P. R. M., Mainzer, A. K., et al. 2010, AJ, 140, 1868 